\title{
Blow up points and the Morse indices of solutions to the Liouville equation in two-dimension
}

\author{
Futoshi Takahashi
}

\begin{tabular}{|c|l|}
\hline Citation & OCAMI Preprint Series \\
\hline Issue Date & 2010 \\
\hline Type & Preprint \\
\hline Textversion & Author \\
\hline Rights & For personal use only. No other uses without permission. \\
\hline Relation & $\begin{array}{l}\text { The following article has been submitted to Advanced Nonlinear Studies. After it } \\
\text { is published, it will be found at } \underline{\text { https://doi.org/10.1515/ans-2012-0107 . }}\end{array}$ \\
\hline
\end{tabular}

From: Osaka City University Advanced Mathematical Institute http://www.sci.osaka-cu.ac.jp/OCAMI/publication/preprint/preprint.html 


\title{
Blow up points and the Morse indices of solutions to the Liouville equation in two-dimension
}

\author{
Futoshi Takahashi \\ Department of Mathematics, Osaka City University \\ \& Osaka City University Advanced Mathematical Institute \\ Sumiyoshi-ku, Osaka, 558-8585, Japan \\ Tel: $(+81)(0) 6-6605-2508$ \\ E-mail: futoshi@sci.osaka-cu.ac.jp
}

Abstract. We consider the Liouville equation

$$
-\Delta u=\lambda e^{u} \quad \text { in } \Omega, \quad u=0 \quad \text { on } \partial \Omega,
$$

on a smooth bounded domain $\Omega$ in $\mathbb{R}^{2}$, where $\lambda>0$ is a parameter. Let $\left\{u_{n}\right\}$ be an m-point blowing up solution sequence of the problem for $\lambda=\lambda_{n} \downarrow 0$, which satisfies

$$
\lambda_{n} \int_{\Omega} e^{u_{n}} d x \rightarrow 8 \pi m \text { as } n \rightarrow \infty
$$

for $m \in \mathbb{N}$. We prove that the number of blow up points $m$ is less than or equal to the Morse index of $u_{n}$ for $n$ sufficiently large.

As a corollary, we show that if a solution $u_{n}$ of Morse index one has the property that $\lambda_{n} \int_{\Omega} e^{u_{n}} d x=O(1)$, then the number of blow up points of the sequence is exactly one. Note that in the last result, we do not need any geometrical assumption such as the convexity of the domain.

Keywords: Morse index, blow up point, Liouville equation.

2010 Mathematics Subject Classifications: 35B40, 35J25.

\section{Introduction and Results.}

In this note we consider the problem

$$
\begin{cases}-\Delta u=\lambda e^{u} & \text { in } \Omega, \\ u=0 & \text { on } \partial \Omega\end{cases}
$$


where $\Omega$ is a smooth bounded domain in $\mathbb{R}^{2}$ and $\lambda>0$. The maximum principle implies that any solution is positive in $\Omega$.

This kind of problem with exponential nonlinearity appears in many fields of mathematics, such as the study of the prescribed Gauss curvature equation ([4] [5]), Chern-Simons gauge theories ([16] [3]), the vortex theory for the turbulent Euler flow ([2]), and so on, and it has attracted many authors for more than decades.

Let $\left\{\lambda_{n}\right\}$ be a sequence of positive numbers satisfying $\lambda_{n} \rightarrow 0$ as $n \rightarrow \infty$. We are interested in a solution sequence $\left\{u_{n}\right\}$ for $\lambda=\lambda_{n}$, which satisfies

$$
\limsup _{n \rightarrow \infty} \lambda_{n} \int_{\Omega} e^{u_{n}} d x \leq C
$$

for some $C<+\infty$. For the solution sequence $\left\{u_{n}\right\}$ satisfying (1.2), Nagasaki and Suzuki [15] showed that there exists a subsequence (which will be denoted by $\left\{u_{n}\right\}$ again) such that

$$
\lambda_{n} \int_{\Omega} e^{u_{n}} d x \rightarrow 8 \pi m \quad(n \rightarrow \infty) \quad \text { for some } m \in \mathbb{N} \cup\{0\} .
$$

Furthermore, if $m=0$, then $u_{n} \rightarrow 0$ uniformly on $\Omega$, and if $m \in \mathbb{N}$, then there exists a set of $m$-points $\mathcal{S}=\left\{a_{1}, \cdots, a_{m}\right\}$ such that each $a_{i}$ is an interior point of $\Omega,\left\|u_{n}\right\|_{L^{\infty}(K)}=O(1)$ for any compact set $K \subset \bar{\Omega} \backslash \mathcal{S},\left.u_{n}\right|_{\mathcal{S}} \rightarrow+\infty$, and

$$
u_{n} \rightarrow 8 \pi \sum_{i=1}^{m} G\left(\cdot, a_{i}\right) \quad \text { in } C_{l o c}^{2}(\bar{\Omega} \backslash \mathcal{S})
$$

as $n \rightarrow \infty$. Here, $G=G(x, y)$ is the Green function of $-\Delta$ under the Dirichlet boundary condition with a pole $y \in \Omega$. See also [1] and [14] for another proof of this fact. In the above sense, we call $\mathcal{S}$ the blow up set for the solution sequence $\left\{u_{n}\right\}$ and each $a_{i} \in \mathcal{S}$ a blow up point of $\left\{u_{n}\right\}$.

In the following, let $i_{M}(u)$ denote the Morse index of a solution $u$ of (1.1), that is, the number of negative eigenvalues of the linearized operator $L_{u}=-\Delta-\lambda e^{u}$. acting on $H_{0}^{1}(\Omega)$.

Now, main result in this note is as follows.

Theorem 1 Let $\left\{u_{n}\right\}$ be a solution sequence of (1.1) for $\lambda=\lambda_{n}$ satisfying (1.3). Then $m \leq i_{M}\left(u_{n}\right)$ for $n$ sufficiently large.

As a corollary, we obtain the following assertion. 
Corollary 2 Let $\left\{u_{n}\right\}$ be a solution sequence of (1.1) for $\lambda=\lambda_{n}$ satisfying (1.2). Assume the Morse index $i_{M}\left(u_{n}\right)=1$ for any $n$ large. Then we have

$$
\lambda_{n} \int_{\Omega} e^{u_{n}} d x \rightarrow 8 \pi, \quad \text { as } n \rightarrow \infty
$$

that is, the number of blow up points of $\left\{u_{n}\right\}$ is exactly 1 .

We remark here that, the number of blow up points of any blowing-up solution sequence to (1.1) is exactly 1 on convex domains. This nonexistence of multiple blow up points on convex domains holds true for a wider class of semilinear problems with blowing-up or concentration phenomena; see [10]. Here, in Corollary 2, we do not need any geometrical assumptions such as the convexity of the domain.

As for the Morse index of solutions, Gladiali and Grossi [9] proved that if $\left\{u_{n}\right\}$ is a solution sequence to (1.1) with $\lambda_{n} \int_{\Omega} e^{u_{n}} d x \rightarrow 8 \pi$, then $i_{M}\left(u_{n}\right)$ is 1 or 2 on any smooth bounded domain $\Omega$. If $\Omega$ is convex, then $i_{M}\left(u_{n}\right)=1$ for $n$ sufficiently large: see Corollary 2.8 in [9].

Related results can be seen in the papers by El Mehdi and Pacella [7] for higher-dimensional cases and [17] for another problem in two dimension.

\section{Proof of Theorem 1.}

In this section, we prove Theorem 1. Let $u_{n}$ be a solution to (1.1) for $\lambda=\lambda_{n}$ with the assumption (1.3). If $m=0$, we have nothing to prove.

In the following, we treat the case $m \in \mathbb{N}$. By the result of [15], we have the blow up set $\mathcal{S}=\left\{a_{1}, \cdots, a_{m}\right\}$, each $a_{i}$ an interior point of $\Omega$. Then we have a sufficiently small $\rho>0$ and $m$ sequences of points $\left\{x_{n}^{i}\right\}$ such that for each $a_{i} \in \mathcal{S}$,

$$
u_{n}\left(x_{n}^{i}\right)=\max _{B_{\rho}\left(x_{n}^{i}\right)} u_{n}(x) \rightarrow \infty, \quad x_{n}^{i} \rightarrow a_{i}(i=1, \cdots, m),
$$

as $n \rightarrow \infty$. See, for example, Li-Shafrir [12].

Let $x_{n}^{i}$ be the above local maximum point of $u_{n}$ around $a_{i} \in \mathcal{S}$. Define the positive number $\delta_{n}^{i}$ and the scaled function $\tilde{u}_{n}^{i}$ by

$$
\begin{aligned}
& \left(\delta_{n}^{i}\right)^{2} \lambda_{n} e^{u_{n}\left(x_{n}^{i}\right)}=1, \\
& \tilde{u}_{n}^{i}(y)=u_{n}\left(\delta_{n}^{i} y+x_{n}^{i}\right)-u_{n}\left(x_{n}^{i}\right), \quad y \in B_{\rho / \delta_{n}^{i}}(0)
\end{aligned}
$$


for $i \in\{1, \cdots, m\}$.

Now we recall the sup + inf type estimate for the blowing-up solutions to (1.1): For a fixed $\rho \in(0,1)$, there exists a constant $C>0$ independent of $i=1, \cdots, m$ and $\lambda_{n}>0$ such that

$$
\left|u_{n}(x)-\log \frac{e^{u_{n}\left(x_{n}^{i}\right)}}{\left(1+\frac{\lambda_{n}}{8} e^{u_{n}\left(x_{n}^{i}\right)}\left|x-x_{n}^{i}\right|^{2}\right)^{2}}\right| \leq C \quad \text { for } x \in B_{\rho}\left(x_{n}^{i}\right)
$$

holds true. See YanYan Li [11], and also [13] for an alternative proof.

We claim that $\delta_{n}^{i}=o(1)$ for any $i=\{1, \cdots, m\}$ as $n \rightarrow \infty$. Indeed, since $u_{n}$ is uniformly bounded for $x \in \partial B_{R}\left(x_{n}^{i}\right)$ for small $R>0$, we have

$$
\left|\log \frac{e^{u_{n}\left(x_{n}^{i}\right)}}{\left(1+\frac{\lambda_{n}}{8} e^{u_{n}\left(x_{n}^{i}\right)} R^{2}\right)^{2}}\right|=O(1)
$$

by the sup + inf estimate (2.3). Since

$$
\frac{e^{u_{n}\left(x_{n}^{i}\right)}}{\left(1+\frac{\lambda_{n}}{8} e^{u_{n}\left(x_{n}^{i}\right)} R^{2}\right)^{2}}=\frac{1}{\left(e^{-\frac{1}{2} u_{n}\left(x_{n}^{i}\right)}+\frac{\lambda_{n}^{1 / 2}}{8}\left(\delta_{n}^{i}\right)^{-1} R^{2}\right)^{2}}
$$

by the definition (2.1), this implies there exist constants $c, C>0$ such that $c \leq \lambda_{n}^{1 / 2}\left(\delta_{n}^{i}\right)^{-1} \leq C$. Thus we have the claim.

Note that $\tilde{u}_{n}^{i}$ satisfies

$$
\left\{\begin{array}{l}
-\Delta \tilde{u}_{n}^{i}=e^{\tilde{u}_{n}^{i}} \quad \text { in } B_{\rho / \delta_{n}^{i}}(0), \\
\tilde{u}_{n}^{i}(0)=0, \tilde{u}_{n}^{i}(x) \leq 0, \quad \forall x \in B_{\rho / \delta_{n}^{i}}(0), \\
\int_{B_{\rho / \delta_{n}^{i}}(0)} e^{\tilde{u}_{n}^{i}} d y=O(1), \quad(n \rightarrow \infty) .
\end{array}\right.
$$

Here, we have used the assumption (1.3).

Next, we claim that

$$
\tilde{u}_{n}^{i} \rightarrow U(y)=-2 \log \left(1+\frac{|y|^{2}}{8}\right)
$$

in $C_{l o c}^{1}\left(\mathbb{R}^{2}\right)$ as $n \rightarrow \infty$ (without choosing a subsequence).

In fact, let $\left\{\tilde{u}_{n(k)}^{i}\right\}$, denoted by $\left\{\tilde{u}_{k}^{i}\right\}$, be any subsequence of $\left\{\tilde{u}_{n}^{i}\right\}$. A result of Brezis-Merle ([1]:Theorem 3) implies that there exists a subsequence of $\left\{\tilde{u}_{k}^{i}\right\}$, still denoted by the same symbol, satisfying one of the following alternatives as $k \rightarrow \infty$ : 
(1) $\tilde{u}_{k}^{i}$ is bounded in $L_{l o c}^{\infty}\left(B_{\rho / \delta_{k}^{i}}(0)\right)$, or

(2) $\tilde{u}_{k}^{i} \rightarrow-\infty$ uniformly on compact sets in $B_{\rho / \delta_{k}^{i}}(0)$, or

(3) there exists a sequence of points $y_{k}^{i} \in B_{\rho / \delta_{k}^{i}}(0)$ such that $\tilde{u}_{n}^{i}\left(y_{k}^{i}\right) \rightarrow+\infty$.

However, by the fact $\tilde{u}_{k}^{i}(0)=\max _{B_{\rho / \delta_{k}^{i}}(0)} \tilde{u}_{k}^{i}(x)=0$, (2) or (3) in the above alternatives cannot happen. Therefore $\left\{\tilde{u}_{k}^{i}\right\}$ is bounded in $L_{l o c}^{\infty}\left(\mathbb{R}^{2}\right)$ along a subsequence, and standard elliptic regularity yields that there exists a subsequence $\left\{\tilde{u}_{k(l)}^{i}\right\}$, denoted by $\left\{\tilde{u}_{l}^{i}\right\}$, and a function $U$ such that $\tilde{u}_{l}^{i} \rightarrow U$ as $l \rightarrow \infty$ in $C_{l o c}^{1}\left(\mathbb{R}^{2}\right)$. Passing to a limit in (2.4) and using Fatou's lemma, we see that $U$ is a solution of

$$
\left\{\begin{array}{l}
-\Delta U=e^{U} \quad \text { in } \mathbb{R}^{2} \\
U(0)=0, U(y) \leq 0, \quad \forall y \in \mathbb{R}^{2} \\
\int_{\mathbb{R}^{2}} e^{U} d y<+\infty
\end{array}\right.
$$

Thus by a result of Chen and $\mathrm{Li}[6]$, we have $U(y)=-2 \log \left(1+\frac{|y|^{2}}{8}\right)$, and this uniqueness of the limit proves the claim. Note that in terms of $\tilde{u}_{n}^{i}$ in (2.2), the estimate (2.3) can be written as

$$
\left|\tilde{u}_{n}^{i}(y)-U(y)\right| \leq C \quad \text { for } y \in B_{\frac{\rho}{\delta_{n}^{i}}}(0), \quad i=1, \cdots, m .
$$

Here, we define two elliptic operators

$$
\begin{aligned}
& L_{n}:=-\Delta_{x}-\lambda_{n} e^{u_{n}} \cdot: H_{0}^{1}\left(B_{\rho}\left(x_{n}^{i}\right)\right) \rightarrow H^{-1}\left(B_{\rho}\left(x_{n}^{i}\right)\right), \\
& \tilde{L}_{n}^{i}:=-\Delta_{y}-e^{\tilde{u}_{n}^{i}} \cdot: H_{0}^{1}\left(B_{\rho / \delta_{n}^{i}}(0)\right) \rightarrow H^{-1}\left(B_{\rho / \delta_{n}^{i}}(0)\right) .
\end{aligned}
$$

The operators (2.5) and (2.6) are related to each other by the formula

$$
\left.\left(\delta_{n}^{i}\right)^{2} L_{n}\right|_{u_{n}(x)=\tilde{u}_{n}^{i}(y)+u_{n}\left(x_{n}^{i}\right)}=\tilde{L}_{n}^{i},
$$

where $x=\delta_{n}^{i} y+x_{n}^{i}$ for $x \in B_{\rho}\left(x_{n}^{i}\right)$ and $y \in B_{\rho / \delta_{n}^{i}}(0)$. Also for a domain $D \subset B_{\rho}\left(x_{n}^{i}\right)$, we have

$$
\left(\delta_{n}^{i}\right)^{2} \lambda_{j}\left(L_{n}, D\right)=\lambda_{j}\left(\tilde{L}_{n}^{i}, D_{n}^{i}\right), \quad D_{n}^{i}=\frac{D-x_{n}^{i}}{\delta_{n}^{i}},
$$

where $\lambda_{j}\left(L_{n}, \underset{\tilde{L}}{D}\right), \lambda_{j}\left(\tilde{L}_{n}^{i}, D_{n}^{i}\right)(j \in \mathbb{N})$ denote the $j$-th eigenvalue of elliptic operators $L_{n}, \tilde{L}_{n}^{i}$ acting on $H_{0}^{1}(D), H_{0}^{1}\left(D_{n}^{i}\right)$ respectively.

We prove 
Lemma 3 There exists $R>0$ such that $\lambda_{1}\left(L_{n}, B\left(x_{n}^{i}, \delta_{n}^{i} R\right)\right)<0$ for n large and for any $i \in\{1, \cdots, m\}$. Here $\left.B\left(x_{n}^{i}, \delta_{n}^{i} R\right)\right)$ denotes an open ball with center $x_{n}^{i}$ and radius $\delta_{n}^{i} R$. Furthermore, these $m$ balls are disjoint for $n$ large.

Proof. Define

$$
w_{n}^{i}(x)=\left(x-x_{n}^{i}\right) \cdot \nabla u_{n}(x)+2, \quad x \in B_{\rho}\left(x_{n}^{i}\right) .
$$

Then $w_{n}^{i}$ satisfies the equation $-\Delta w_{n}^{i}(x)=\lambda_{n} e^{u_{n}} w_{n}^{i}, x \in B_{\rho}\left(x_{n}^{i}\right)$, and if we define $\tilde{w}_{n}^{i}$ as

$$
\tilde{w}_{n}^{i}(y)=w_{n}^{i}\left(\delta_{n}^{i} y+x_{n}^{i}\right)=y \cdot \nabla \tilde{u}_{n}(y)+2, \quad y \in B_{\rho / \delta_{n}^{i}}(0),
$$

then $\tilde{w}_{n}^{i}$ satisfies $-\Delta \tilde{w}_{n}^{i}(y)=e^{\tilde{u}_{n}^{i}} \tilde{w}_{n}^{i}(y)$ for $y \in B_{\rho / \delta_{n}^{i}}(0)$. Since $\tilde{u}_{n}^{i} \rightarrow U$ in $C_{l o c}^{1}\left(\mathbb{R}^{2}\right)$, we have

$$
\tilde{w}_{n}^{i}(y) \rightarrow y \cdot \nabla U+2=2 \frac{8-|y|^{2}}{8+|y|^{2}}<0 \quad \text { if }|y|=R>2 \sqrt{2}
$$

as $n \rightarrow \infty$. Set $A_{n}^{i}=\left\{y \in B(0, R): \tilde{w}_{n}^{i}(y)>0\right\}, A_{n}^{i} \neq \phi$, and define

$$
\bar{w}_{n}^{i}(y)= \begin{cases}\tilde{w}_{n}^{i}(y) & y \in A_{n}^{i}, \\ 0 & y \in B(0, R) \backslash \overline{A_{n}^{i}}\end{cases}
$$

If we test

$$
\lambda_{1}\left(\tilde{L}_{n}^{i}, B(0, R)\right)=\inf _{v \in H_{0}^{1}(B(0, R))} \frac{\int_{B(0, R)}|\nabla v|^{2} d y-\int_{B(0, R)} e^{\tilde{u}_{n}} v^{2} d y}{\int_{B(0, R)} v^{2} d y}
$$

by $\bar{w}_{n}^{i} \in H_{0}^{1}(B(0, R))$, we see that $\lambda_{1}\left(\tilde{L}_{n}^{i}, B(0, R)\right) \leq 0$. Actually, we have $\lambda_{1}\left(\tilde{L}_{n}^{i}, B(0, R)\right)<0$, since if equality holds, $\bar{w}_{n}^{i}$ would be the first eigenfunction of $\tilde{L}_{n}^{i}$ on $B(0, R)$, which must be strictly positive. But this contradicts to the fact that $\bar{w}_{n}^{i}$ is 0 near $\partial B(0, R)$. Finally, scaling formula (2.7) proves the first half part of the Lemma.

Next, we claim that $B\left(x_{n}^{i}, \delta_{n}^{i} R\right)$ and $B\left(x_{n}^{j}, \delta_{n}^{j} R\right)$ are disjoint for $n$ large, if $i \neq j$. Indeed, since $\tilde{u}_{n}^{i} \rightarrow U, \tilde{u}_{n}^{j} \rightarrow U$ in $C_{l o c}^{1}\left(\mathbb{R}^{2}\right), U(y)=-2 \log \left(1+|y|^{2} / 8\right)$ is strictly concave and $y \cdot \nabla \tilde{u}_{n}(y) \rightarrow y \cdot \nabla U(y)<0$ on $B(0, R) \backslash\{0\}, u_{n}$ is also strictly concave and $\left(x-x_{n}^{i}\right) \cdot \nabla u_{n}(x)<0$ on $B\left(x_{n}^{i}, \delta_{n}^{i} R\right) \backslash\left\{x_{n}^{i}\right\}$ for $n$ large. 
In particular, there is not a critical point in $B\left(x_{n}^{i}, \delta_{n}^{i} R\right)$ other than $\left\{x_{n}^{i}\right\}$. The same holds for $u_{n}$ on $B\left(x_{n}^{j}, \delta_{n}^{j} R\right) \backslash\left\{x_{n}^{j}\right\}$, and this concavity property proves the claim.

Now by Lemma 3 , we have $m$ open balls $B^{1}, \cdots, B^{m}, B^{i}=B\left(x_{n}^{i}, \delta_{n}^{i} R\right)$, which are disjoint, and

$$
\lambda_{1}\left(L_{n}, B^{i}\right)<0 \quad \text { for } i=1, \cdots, m .
$$

Furthermore, by a variational characterization of $m$-th eigenvalue of $L_{n}$, we see that

$$
\lambda_{m}\left(L_{n}, \Omega\right) \leq \sum_{i=1}^{m} \lambda_{1}\left(L_{n}, B^{i}\right) .
$$

Though this fact is well-known, we give a proof in Appendix for the reader's convenience.

From (2.9) and (2.10), we have $\lambda_{m}\left(L_{n}, \Omega\right)<0$. On the other hand, by the definition of the Morse index of $u_{n}$, we have $\lambda_{i_{M}\left(u_{n}\right)+1}\left(L_{n}, \Omega\right) \geq 0$. Therefore $m \leq i_{M}\left(u_{n}\right)$, and this proves Theorem 1 .

Proof of Corollary. Let $\left\{u_{n}\right\}$ be a solution sequence as in Corollary 2, and choose any subsequence $\left\{u_{n(k)}\right\}$ of $\left\{u_{n}\right\}$, denoted by $\left\{u_{k}\right\}$. By a result of [15], there exists a subsequence $\left\{u_{k(l)}\right\}$ of $\left\{u_{k}\right\}$, denoted by $\left\{u_{l}\right\}$, such that $\lambda_{l} \int_{\Omega} e^{u_{l}} d x \rightarrow 8 \pi m$ for some $m \in \mathbb{N} \cup\{0\}$. By Theorem 1, we have $m \leq i_{M}\left(u_{l}\right) \equiv 1$. If $m=0$, then $u_{l}$ coincides with the minimal solution for $l$ large, and since the Morse index of the minimal solution is 0 , this cannot happen. Thus we have $m=1$ and $\lambda_{l} \int_{\Omega} e^{u_{l}} d x \rightarrow 8 \pi$ as $l \rightarrow \infty$. Since $\left\{u_{k}\right\}$ is arbitrarily chosen, we have

$$
\lambda_{n} \int_{\Omega} e^{u_{n}} d x \rightarrow 8 \pi \quad \text { as } n \rightarrow \infty
$$

for the full sequence $\left\{u_{n}\right\}$.

\section{Appendix.}

In this appendix, we prove the following lemma, which gives the validity of $(2.10)$. 
Lemma 4 Let $L=-\Delta-c(x)$. denote a second-order elliptic differential operator acting on $H_{0}^{1}(\Omega)$, where $\Omega \subset \mathbb{R}^{N}(N \geq 1)$ is a smooth bounded domain and $c \in C(\bar{\Omega})$. If $D_{1}, \cdots, D_{m}$ are disjoint smooth subdomains of $\Omega$, then we have

$$
\lambda_{m}(L, \Omega) \leq \sum_{i=1}^{m} \lambda_{1}\left(L, D_{i}\right)
$$

where $\lambda_{j}(L, D)$ denote the $j$-th eigenvalue of $L$ acting on $H_{0}^{1}(D)$ (counted with multiplicities) for $D \subset \Omega$.

Proof. Let $\psi_{i} \in H_{0}^{1}\left(D_{i}\right)(i=1, \cdots, m)$ be the first eigenfunction of $L$ on $D_{i}$ :

$$
\begin{cases}L \psi_{i}=\lambda_{1}\left(L, D_{i}\right) \psi_{i} & \text { in } D_{i} \\ \psi_{i}=0 & \text { on } \partial D_{i}\end{cases}
$$

normalized as $\left\|\psi_{i}\right\|_{L^{2}\left(D_{i}\right)}=1$. We can extend $\psi_{i}$ as 0 outside $D_{i}$ and obtain new function in $H_{0}^{1}(\Omega)$, which will be denoted again by $\psi_{i}$. Set $u=\sum_{i=1}^{m} \alpha_{i} \psi_{i}$ for $\alpha_{i} \in \mathbb{R}, u \in H_{0}^{1}(\Omega)$. Since

$$
\begin{aligned}
& \|u\|_{L^{2}(\Omega)}^{2}=\sum_{i=1}^{m} \alpha_{i}^{2}\left\|\psi_{i}\right\|_{L^{2}\left(D_{i}\right)}^{2}=\sum_{i=1}^{m} \alpha_{i}^{2}, \\
& (u, \phi)_{L^{2}(\Omega)}=\sum_{i=1}^{m} \alpha_{i}\left(\psi_{i}, \phi\right)_{L^{2}\left(D_{i}\right)}
\end{aligned}
$$

for any $\phi \in H_{0}^{1}(\Omega)$, we have $\left(\alpha_{1}, \cdots, \alpha_{m}\right) \neq(0, \cdots, 0)$ such that

$$
\|u\|_{L^{2}(\Omega)}^{2}=1, \quad \text { and } \quad\left(u, \phi_{j}\right)_{L^{2}(\Omega)}=0 \quad(j=1, \cdots, m-1)
$$

holds true, where $\phi_{j}$ denotes the $j$-th eigenfunction corresponding to $\lambda_{j}(L, \Omega)$. By a variational characterization of $\lambda_{m}(L, \Omega)$, we have

$$
\begin{aligned}
\lambda_{m}(L, \Omega)=\inf \left\{(L v, v)_{L^{2}(\Omega)}\right. & \|v\|_{L^{2}(\Omega)}=1, \\
& \left.\left(v, \phi_{j}\right)_{L^{2}(\Omega)}=0(j=1, \cdots, m-1)\right\} .
\end{aligned}
$$

Testing this by $u=\sum_{i=1}^{m} \alpha_{i} \psi_{i}$, we obtain

$$
\begin{aligned}
& \lambda_{m}(L, \Omega) \leq(L u, u)_{L^{2}(\Omega)}=\left(\sum_{i=1}^{m} \alpha_{i} L \psi_{i}, \sum_{i=1}^{m} \alpha_{i} \psi_{i}\right)_{L^{2}(\Omega)} \\
& =\sum_{i=1}^{m} \alpha_{i}^{2} \lambda_{1}\left(L, D_{i}\right)\left\|\psi_{i}\right\|_{L^{2}\left(D_{i}\right)}^{2} \leq \sum_{i=1}^{m} \lambda_{1}\left(L, D_{i}\right) .
\end{aligned}
$$


Acknowledgments. The author would like to thank Massimo Grossi for invaluable discussions on the subject in this paper. Part of this work was supported by JSPS Grant-in-Aid for Scientific Research (C), No. 20540216.

\section{References}

[1] H. Brezis, and F. Merle: Uniform estimates and blow-up behavior for solutions of $-\Delta u=V(x) e^{u}$ in two dimensions, Comm. Partial Differential Equations 16 (1991) 1223-1253.

[2] E. Caglioti, P.L. Lions, C. Marchioro, and M. Pulvirenti: A special class of stationary flows for two-dimensional Euler equations: a statistical mechanics description, I \& II. Comm. Math. Phys. 143 (1992) 501-525 \& 174 (1995) 229-260.

[3] D. Chae, and O. Imanuvilov: The existence of non-topological multivortex solutions in the relativistic self-dual Chern-Simons theory, Comm. Math. Phys. 215 (2000) 119-142.

[4] S.Y.A. Chang, and P.C. Yang: Prescribing Gaussian curvature on $S^{2}$, Acta Math. 159 (1987) 215-259.

[5] S.Y.A. Chang, and P.C. Yang: Conformal deformation of metrics on $S^{2}$, J. Differential Geom. 27 (1988) 259-296.

[6] E. Chen, and C. Li: Classification of solutions of some nonlinear elliptic equations, Duke Math. J. 63(3) (1991) 615-622.

[7] K. El Mehdi, and F. Pacella: Morse index and blow-up points of solutions of some nonlinear problems, Atti. Accad. Naz. Lincei Mat. Appl. 13 (2002) 101-105.

[8] F. Gladiali, and M. Grossi: Some results on the Gel'fand problem, Comm. Partial Differential Equations 29 (2004) 1335-1364.

[9] F. Gladiali, and M. Grossi: On the spectrum of a nonlinear planar problem, Ann. I. H. Poincaré 26 (2009) 191-222. 
[10] M. Grossi, and F. Takahashi: Nonexistence of multi-bubble solutions to some elliptic equations on convex domains, J. Funct. Anal. 259 (2010) 904-917.

[11] Y. Y. Li: Harnack type inequality: the method of moving planes, Commun. Math. Phys. 200 (1999) 421-444.

[12] Y. Y. Li, and I. Shafrir: Blow-up analysis for solutions of $-\Delta u=V e^{u}$ in dimension two, Indiana Univ. Math. J. 43 (1994) 1255-1270.

[13] C. S. Lin: An expository survey on recent development of mean field equations, Discrete Contin. Dyn. Syst. 19 no.2, (2007) 387-410.

[14] L. Ma, and J. Wei: Convergence for a Liouville equation, Comment. Math. Helv. 76 (2001) 506-514.

[15] K. Nagasaki, and T. Suzuki: Asymptotic analysis for two-dimensional elliptic eigenvalue problems with exponentially dominated nonlinearities, Asymptotic Anal. 3 (1990) 173-188.

[16] M. Struwe, and G. Tarantello: On multivortex solutions in ChernSimons gauge theories, Boll. Unione Mat. Ital. Sez. B. 8 (1998) 109-121.

[17] F. Takahashi: Morse indices and the number of maximum points of some solutions to a two-dimensional elliptic problem, Archiv der Math. 93 (2009) 191-197. 